Dialectologia 22 (2019), 57-80.

ISSN: 2013-2247

Received 23 June 2017.

Accepted 3 October 2017.

\title{
DOS PATRONES DEL LEÍSMO EN EL ESPANOL DE AZOGUES, ECUADOR: UN ANALISIS BASADO EN DATOS RECOGIDOS DE FACEBOOK MESSENGER
}

\author{
Jordan LAVENDER \\ Promfet School* \\ jlavender@pomfret.org
}

\section{Resumen}

Este estudio analiza el leísmo de 3a persona y el leísmo de cortesía en los mensajes de Facebook Messenger de hablantes ecuatorianos de la ciudad de Azogues, Ecuador. Analiza el efecto de factores lingüísticos y extralingüísticos en cuanto a su uso en el habla de monolingües en español. El leísmo de 3ạ persona y el leísmo de cortesía ocurren con más frecuencia que su equivalente con la forma estándar, aun más para el leísmo de cortesía. Los informantes prefieren el leísmo con referentes animados y lo(s) con referentes no animados y el leísmo de cortesía acompaña a referentes femeninos singulares con mucha frecuencia. Los dos tipos de leísmo se comportan igual en cuanto a su preferencia para una posición preverbal en la oración. Los informantes con educación secundaria evitan el leísmo de 3a persona pero no evitan el leísmo de cortesía.

\section{Palabras clave}

lingüística de contacto, leísmo, variación, sociolingüística, comunicación mediada por computadoras (CMC)

* Pomfret School, 398 Pomfret St., Pomfret Center, CT 06259, USA. 
TWO LEÍSMO PATTERNS IN AZOGUES, ECUADOR: AN ANALYSIS OF DATA COLLECTED FROM FACEBOOK MESSENGER

Abstract

This study analyzes both leísmo and leísmo de cortesía in the Facebook Messenger messages of Ecuadorian speakers from Azogues, Ecuador. It examines the effect of linguistic and extralinguistic factors with regard to the use of leísmo by monolingual Spanish speakers from this region. Both leísmo and leísmo de cortesía occur more frequently than their standard equivalents, even more so for leísmo de cortesía. Informants prefer to use leísmo with both animate referents and non-animate referents. Leísmo de cortesía is used more frequently with feminine, singular referents. Both types of leísmo behave equally with regard to their preference in preverbal positions in sentences. Informants with secondary education avoid leísmo but show no avoidance of leísmo de cortesía.

\section{Keywords}

contact linguistics, leismo, variation, sociolinguistics, computer-mediate communication (CMC)

\section{Objetivos e hipótesis de partida}

Este estudio analiza dos patrones del leísmo encontrados en el español de Azogues, Ecuador: el leísmo de 3a persona y el leísmo de cortesía o de 2a persona. El leísmo refiere al uso de $l e(s)$ en vez de $l o(s), l a(s)$ y se usa cuando se refiere a la $2 \underline{a}$ persona, usted (de cortesía) o en la 3a persona (de persona). Estos dos patrones de leísmo tienen raíces diferentes en cuanto a cómo se explica su origen. Un patrón de leísmo es un producto del contacto entre el español y el quichua y otro es una estrategia utilizada para evitar ambigüedad cuando un hablante refiere a un interlocutor presente en una conversación considerando que $l o(s), l a(s)$ pueden referir a referentes no partícipes en una conversación.

El leísmo no solo se encuentra en el español peninsular, también es un rasgo del español andino del Ecuador (García \& Otheguy 1983; Palacios 2002, 2005) y del Perú (Caravedo 1996; Godenzzi 1986, Klee 1990). Lipski (1994) describe la situación general en el español ecuatoriano.

En buena parte de Ecuador se suele emplear le como clítico de objeto directo para SSNN masculinos singulares, en contra de las tendencias loístas del español 
de America del Sur... Le puede extenderse incluso a los objetos femeninos. Muchos ecuatorianos, tanto bilingües como monolingües, no poseen el clitico la en su repertorio, y, en consecuencia, emplean le (Lipski 1994: 269).

El leísmo de $3^{a}$ persona en el español ecuatoriano es un producto del contacto prolongado entre el español y el quichua (Palacios 2005). Muchos investigadores han observado el uso del leísmo de 3 ạ persona en el habla monolingüe ecuatoriano. El enfoque de investigaciones previas ha sido analizar el leísmo de $3 \underline{a}$ persona en variedades del español hablado por bilingües quechua-español en Ecuador. Por el contrario, este estudio enfoca solo en el habla monolingüe hispanohablante y añade al conocimiento sobre los patrones de leísmo encontrados en el habla de monolingües castellanohablantes.

Este estudio analiza datos recogidos de hablantes pertenecientes a la ciudad de Azogues, en la provincia de Cañar en Ecuador. En diferenciación de otros estudios que se basan en entrevistas sociolingüísticas o en encuestas lingüísticas, este estudio utiliza datos recogidos de Facebook Messenger, los cuales representan datos de conversaciones informales entre usuarios de Facebook Messenger de esta región en Ecuador.

Tiene como meta este estudio entender el leísmo de 3 a persona y el leísmo de cortesía, o de $2^{a}$ persona, en el español hablado en la ciudad de Azogues, Ecuador. Este estudio contesta las siguientes preguntas de investigación.

(1) ¿Cuál es la distribución y frecuencia de leísmo de 3a persona (masculina y femenina) y leísmo de cortesía (usted) en la variedad de español hablada en Azogues, Ecuador?

(2) ¿Cuáles son los factores lingüísticos y extralingüísticos que afectan su uso?

(3) ¿Se comporta el leísmo de cortesía lingüísticamente como el leísmo de 3ạ persona o como otro fenómeno aparte? 


\subsection{El quichua y el español en Ecuador}

En Ecuador hay alrededor de 2 millones de hablantes del quichua. El quichua tiene cierta posición semioficial en el país. Además de eso, en Ecuador, el español y el quichua han estado en contacto por casi 500 años. El quichua era lengua franca en el imperio inca que se hablaba a través de sus territorios. Con la llegada de los españoles, el quichua fue adoptado como lengua indígena de gran parte de la población indígena con el español sirviendo como lengua oficial del gobierno. Muchos sacerdotes y misioneros aprendieron el quichua para apoyar en el proceso de cristianización de la población indígena y produjeron los primeros documentos escritos en quichua. Sin embargo, el quichua y las personas que lo hablaban formaban parte de la clase social baja en el periodo de colonización. Con los movimientos de independencia en Sudamérica, la situación lingüística no cambió en cuanto al estatus del quichua en la sociedad. En el siglo XX hubo movimientos de indigenismo que apoyaron el uso del quichua, pero la fuerza de la historia y las actitudes negativas hacia el quichua y la población indígena siguieron en la cultura popular (Haboud 1998).

Haboud (1998) nota los niveles de bilingüismo en las provincias serranas. La zona quichuahablante se concentra en las provincias centrales al sur de Pichinga. El mapa (Figura 1) demuestra las provincias ecuatorianas. Las provincias de Tungurahua, Bolívar, Cotopaxi y Chimborazo tienen más hablantes del quichua que otras regiones. Entonces, hay ciertas regiones en bloques donde hay más concentración de hablantes nativos de quichua, particularmente en la zona central de la sierra. La única excepción es la región de Imabura en el norte que tiene un nivel bastante alto de quichuahablantes. 


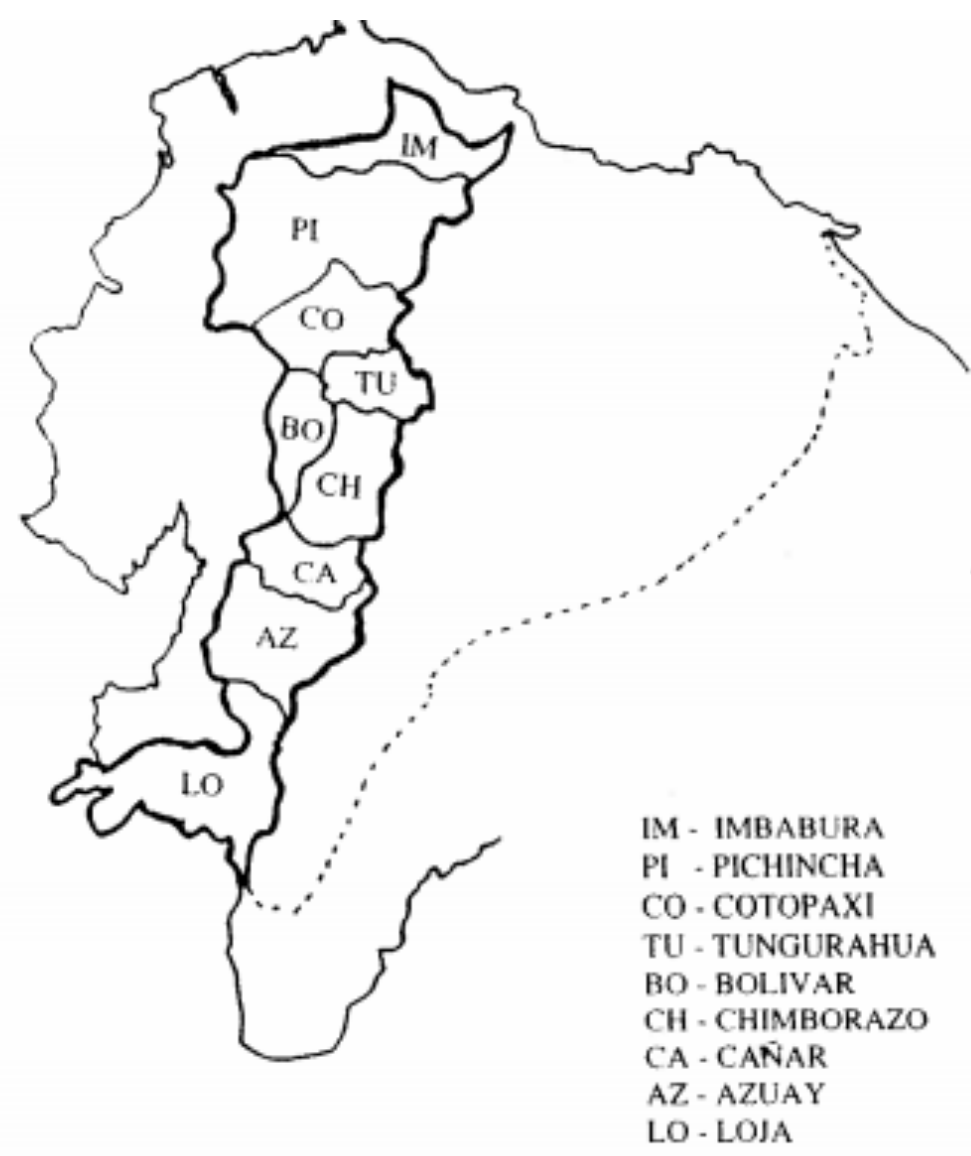

Figura 1. Mapa de las provincias serranas del Ecuador (Haboud 1998: 76)

Hay un continuo de niveles de bilingüismo que se concentra en el centro del país, incluso a unas variedades mezcladas como Media Lengua que se habla en regiones con más bilingües (Muysken 1985). Las provincias sureñas, Azuay y Loja, tienen menos hablantes de quichua. Büttner (1993) nota que hay comunidades bilingües quichuaespañol en Cañar. Sin embargo, la vitalidad del quichua en Cañar está en peligro y solo hay alrededor de 90.000 hablantes. $^{1}$

\section{Variación en el sistema pronominal en español}

El sistema pronominal clítico español distingue caso (acusativo, dativo) y género (masculino, femenino).

\footnotetext{
${ }^{1}$ Información de: http://www.oralidadmodernidad.com/.
} 


\begin{tabular}{|l|l|l|}
\hline & Acusativo & Dativo \\
\hline $2^{\text {da }}$ persona singular & Lo, la & Le \\
\hline $2^{\text {da }}$ persona plural & Los, las & Les \\
\hline $3^{\text {ra }}$ persona singular & Lo, la & Le \\
\hline $3^{\text {ra }}$ persona singular & Los, las & les \\
\hline
\end{tabular}

Tabla 1. El sistema pronominal clítico en el español estándar

García \& Otheguy (1983) describieron la variación pronominal en Quito como una variación entre dos sistemas, uno que es completo con las opciones $(l o, l a, l e)$ y otro que es parcial con las opciones $(l o, l e)$, notando que la extensión de le(s) originó del habla bilingüe pero su uso ha extendido a los monolingües. De la misma forma los dos sistemas se distinguen en cuanto a cómo marcan a los referentes femeninos. En el primer sistema, hay una forma femenina diferenciada, pero, en el segundo sistema, le se utiliza para los objetos directos femeninos y para referentes desconocidos. Por lo tanto, lo que distingue estos dos sistemas es el mantenimiento del género en cuanto a los pronombres clíticos.

Hay patrones de variación en cuanto al sistema de pronombres clíticos en unas variedades del español que manifiesta en la forma del leísmo, lo cual refiere al "uso de la forma le en lugar de lo... como pronombre para referirse al complemento directo" (Fernández-Ordóñez 1999: 1319). El leísmo es un rasgo asociado con el español peninsular. Fernández-Ordóñez (1999: 1319) describe en orden de aceptabilidad de las diferentes posibilidades en cuanto al leísmo en el español peninsular:

1. ¿Conoces a Juan? Sí, le conozco hace tiempo.

2. ¿Sabes dónde está mi libro? No, no le he visto por aquí.

3. Esta tarde voy a recoger a los niños del colegio y les llevaré al parque.

4. Fui a buscar los discos que querías y les encontré en la tienda de abajo.

5. A María hace tiempo que no le veo.

6. Aquí no hay monjas. En la guerra les mataron a todas. 
En el español peninsular, la forma más aceptable es el uso de le para referirse a objetos directos masculinos singulares animados como en el caso del primer ejemplo (1). La forma menos aceptable es el uso de le con objetos directos femeninos plurales, como en (6).

Fernández-Ordóñez (1999) estudió el uso del leísmo en Ecuador, notando que el uso de le(s) se ha generalizado independientemente de función sintáctica o del género. $L e(s)$ generalmente refieren a antecedentes animados, aunque hay la posibilidad de que se puedan referir a antecedentes inanimados también. Este uso de le(s) es un rasgo de hablantes bilingües y monolingües. Sin embargo, los hablantes monolingües tienden a mantener el uso de $l o(s)$, $l a(s)$ más que los bilingües.

Palacios $(2002,2005)$ describió las particularidades del leísmo ecuatoriano. Ella nota que el leísmo es más generalizado en cuanto a su uso con referentes masculinos y femeninos y entre referentes animados e inanimados (Palacios 2002: 390). También menciona que en este sistema, el pronombre $l e(s)$ es la forma generalizada de tercera persona con el resultado de las siguientes opciones no sean marcadas:
a. Le vi (al niño)
b. Le vi (a la niña)
c. Le vi (al libro)
d. Le vi (a la casa) (Palacios 2002: 390)

El español ecuatoriano andino tiende a la simplificación con una preferencia leísta, sin embargo, hay patrones de variación en los hablantes ecuatorianos.

El leísmo de 3a persona en el español ecuatoriano es un tipo de variación que se atribuye al contacto entre quichua y español. Unos aspectos en la gramática del quichua afectan este uso. El quichua no tiene marcadores de género y no tiene género en su sistema gramatical. Tampoco tiene un sistema de marcadores que corresponden al sistema de dativo y acusativo en el español (Palacios 2002: 402). El leísmo de 3a persona empieza en el habla de bilingües y se transfiere al habla de monolingües.

El efecto de factores sociales sobre la diferenciación entre estos dos sistemas sigue siendo no bien estudiado, pero unos investigadores han comentado sobre su 
efecto. García \& Otheguy (1983) notaron que aun los hablantes con educación no utilizan las formas lo, la en una manera estándar. Fernández-Ordóñez (1999) postuló que la educación pudo crear una reducción en el uso de leísmo. Sin embargo, según Palacios (2005), el uso de leísmo en Ecuador no es estigmatizado (Palacios 2005: 67).

El leísmo de cortesía, acuñado por Lorenzo Ramos (1981, 1984), se refiere al uso de le(s) para un objeto directo cuando el referente es usted. Se usa "por el deseo de evitar la ambigüedad de sentido que acarrearía el uso de los pronombres $l o(s) l a(s)$ ya que estos podrían referirse tanto a un interlocutor presente como a una tercera persona no partícipe en la conversación" (RAE 2009: 395). El uso del leísmo de cortesía es aceptable por la RAE en ciertas situaciones, por ejemplo, en fórmulas fijas de saludo o despedida y con referentes masculinos. Branza (2009a, 2009b, 2011) ha estudiado el fenómeno del leísmo de cortesía en muchos lugares en Hispanoamérica y España, notando su uso en Ecuador.

Dumitrescu \& Branza (2012) analizaron el leísmo de cortesía y lo describieron como un cambio en progreso que no ha recibido la atención que merece. Se ha notado en muchas variedades del español como en el Caribe (Vaquero de Ramírez 1996) y México (Cantero Sandoval 1979). Estos investigadores notan su uso habitual en fórmulas fijas con verbos como saludar, invitar, ver, esperar, felicitar, etc. Gómez Torrego (2006) nota que el leísmo de cortesía ha extendido en América incluso con referentes femeninos. Ha llegado a ser un fenómeno panhispánico (Dumitrescu \& Branza 2012).

Hay debate sobre si el leísmo de cortesía es un tipo de "leísmo" o un fenómeno aparte. Demello (2002) no lo considera como una forma de leísmo porque las motivaciones para su uso son sociales, basadas en la noción de que el uso de le es más prestigioso. Fernández-Ordóñez (1999) también analiza el leísmo de cortesía como forma aparte del leísmo de persona debido a su intención de ser un tratamiento de respeto. La RAE (2009) ofrece una posición entre las de Demello (2002) y FernándezOrdóñez (1999) que distingue entre tres tipos de leísmo: (1) el leísmo de persona masculina (A); (2) el leísmo de persona femenina (B); (3) el leísmo de cosa (C). Según esta clasificación, el leísmo de cortesía es "una variante restringida de los tipos A y B" (Fernández-Ordóñez 1999: 1213). 
Dumitrescu \& Branza (2012) analizan el leísmo de cortesía como una extensión de estrategias de cortesía, que tienen como motivo "proteger la imagen negativa del interlocutor y en reforzar su imagen positiva" (Dumitrescu \& Branza 2012: 683). Según este análisis, el leísmo de cortesía en fórmulas fijas, como saludo, despedida o deseos de bienestar, sirve como un tipo de face flattering act (Kerbat-Orecchioni 1997) que tiene un papel importante en una conversación el fortalecimiento de una imagen positiva del interlocutor al que se dirige el hablante (Albelda 2008). Este tipo de face flattering act sirve como una manifestación de solidaridad entre el locutor y el interlocutor (Dumitrescu 2011). Dumitrescu \& Branza (2012) también sugieren que el leísmo de cortesía pueda ser una manera de utilizar lenguaje inclusivo, debido al hecho que le no revela el género del referente. Finalmente, ofrecen una motivación social del uso de leísmo de cortesía. Cuando un hablante acude al leísmo de cortesía "está llevando a cabo implícitamente un trabajo de autoimagen, en el sentido de que quiere presentarse a los ojos de su interlocutor como una persona refinada y educada" (Dumitrescu \& Branza 2012: 684) -un marcador de prestigio.

\section{Metodología}

Como base de datos se observaron las conversaciones en Facebook Messenger de dos participantes ecuatorianas: P1 y P2, que proveyeron mensajes con otros amigos ecuatorianos de sus cuentas de Facebook Messenger. La tabla siguiente demuestra los datos recogidos de cada informante.

\begin{tabular}{|l|l|l|l|}
\hline & $\mathbf{P 1}$ & $\mathbf{P 2}$ & Total \\
\hline Número de amigos en Facebook Messenger & 32 & 12 & 44 \\
\hline Número de palabras constituyendo un corpus & 23.860 & 8.786 & 32.646 \\
\hline Número de mensajes & 2.044 & 573 & 2.617 \\
\hline Número de conversaciones entre amigos & 115 & 30 & 145 \\
\hline
\end{tabular}

Tabla 2. Datos de cada informante 
P1 y P2 son mujeres ecuatorianas de Azogues, Ecuador con educación universitaria. El mapa en Figura 2 demuestra la zona de donde vienen los informantes de este estudio.

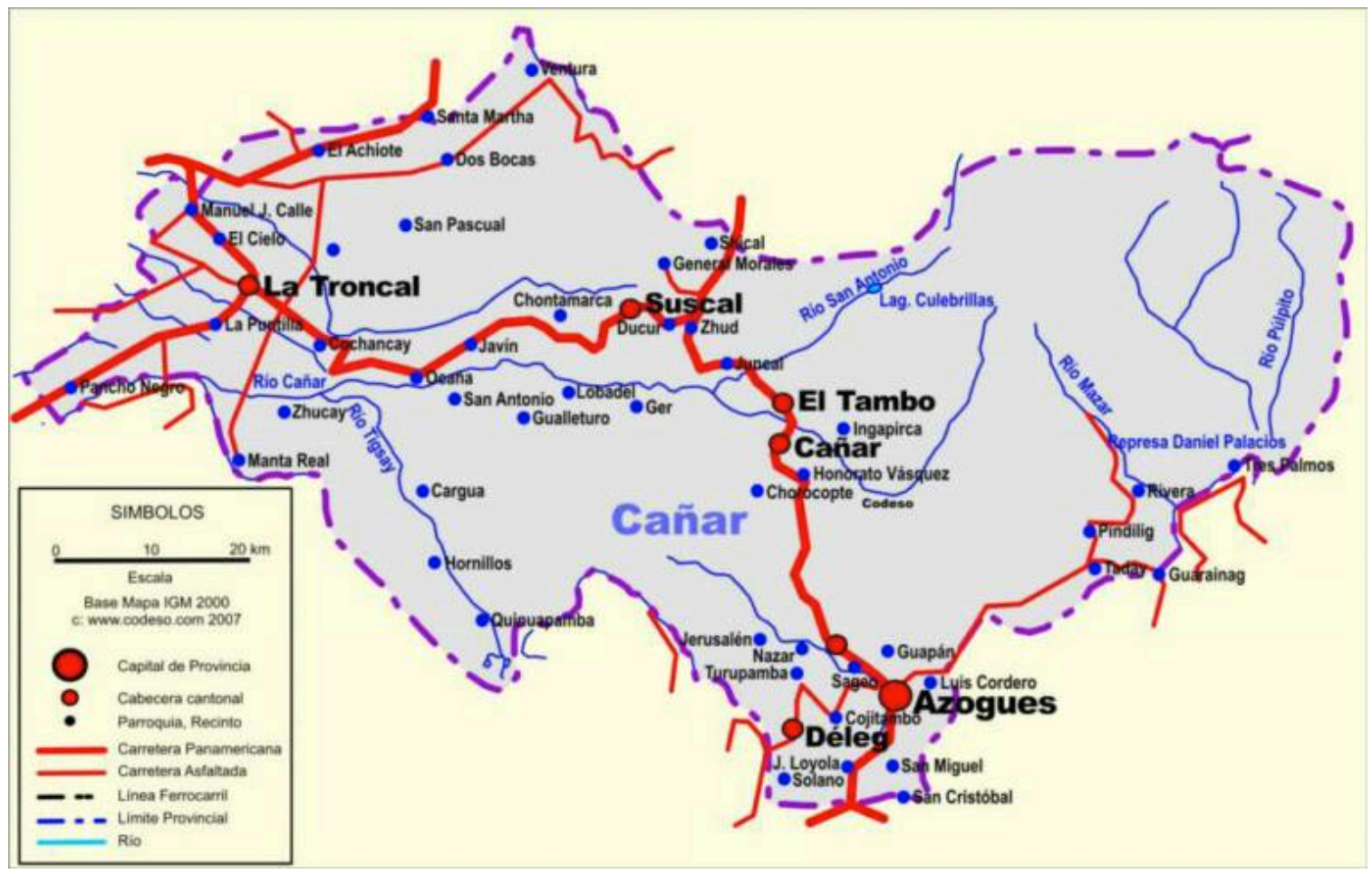

Figura 2. Mapa de Cañar (página web del Gobierno Provincial de Cañar)²

P1 y sus amigos constituyen un grupo de hablantes mayores de 40 años de edad mientras P2 y sus amigos constituyen un grupo de hablantes menores de 40 años de edad. P1 utiliza Facebook Messenger con más frecuencia que P2, entonces el número de mensajes recogidos de sus conversaciones supera los de P2. Este estudio considera el efecto de unos factores lingüísticos y sociales en el uso de leísmo de 3 a persona y leísmo de cortesía. Los factores lingüísticos considerados son: (1) +/- animado; (2) el tipo de referente en cuanto al género y número; (3) la posición de pronombres pre- o posverbal. Los factores sociales considerados son: (1) sexo, y; (2) nivel de educación. Las participantes, P1 y P2, y sus amigos son hablantes nativos de español sin competencia en quichua.

\footnotetext{
${ }^{2}$ http://www.gobiernodelcanar.gob.ec/public html/paginas/mapa-vial.61.
} 
No se analizó la edad como factor extralingüístico que podría afectar el uso de leísmo de cortesía debido a las preferencias en cuanto a la forma de tratamiento de los informantes jóvenes. Entre los amigos de P1 y sus amigos, generalmente se tratan de usted entre sí, con unas excepciones, mientras que entre P2 y sus amigos se tratan de tú o vos pronominal. Unos investigadores han encontrado este patrón en la sierra donde hay vacilación entre el uso de usted entre familias y tuteo o voseo entre amigos (Calderón Campos 2010).

El sistema educativo ecuatoriano consiste en dos niveles: la escuela primaria y la escuela secundaria. Los estudiantes tienen que asistir por obligación constitucional hacia el final del séptimo grado. Es común en la sierra que los estudiantes no completen la secundaria por razones económicas. La mayoría de los informantes asistieron escuelas públicas con la excepción de unos que asistieron a escuelas privadas católicas. Se incluyeron en la designación "Universidad" en la Tabla 3 a los informantes que han completado estudios parciales en la universidad y otros estudios adicionales después de la secundaria. También, se incluyen en esta categoría los que están en progreso de completarlos o los que han completado programas en escuelas técnicas.

La tabla siguiente demuestra la división de amigos de P1 y P2 en cuanto a estos factores extralingüísticos.

\begin{tabular}{|l|l|l|l|l|}
\hline \multicolumn{2}{|l|}{ Factor } & $P 1$ & $P 2$ & Total \\
\hline \multirow{4}{*}{ Sexo } & Masculino & 16 & 7 & 23 \\
\cline { 2 - 5 } & Femenino & 17 & 5 & 22 \\
\hline \multirow{2}{*}{$\begin{array}{l}\text { Estatus socio- } \\
\text { económico }\end{array}$} & Universidad & 9 & 8 & 17 \\
\cline { 2 - 5 } & Secundaria & 13 & 5 & 18 \\
\cline { 2 - 5 } & Primaria & 11 & 0 & 11 \\
\hline Total & 32 & 12 & 44 (incluyendo informantes P1 y P2) \\
\hline
\end{tabular}

Tabla 3. Informantes del estudio

Facebook Messenger es un servicio de mensajería instantánea de Facebook que se puede utilizar independientemente en una aplicación separada. Facebook Messenger funciona como aplicación en computadora o en teléfono celular. Este tipo de CMC (comunicación mediada por computadoras) es un tipo simultáneo porque un usuario 
anticipa una respuesta rápida del interlocutor. Según muchos investigadores, la mensajería instantánea es la forma de CMC más parecida a la conversación natural (Crystal 2006; Sebba 2012). En este sentido, el recoger de datos de Facebook Messenger ofrece una oportunidad de analizar datos recogidos de una fuente informal y sin influencia del investigador.

\section{Resultados}

La tabla número cuatro muestra la distribución y frecuencia de todos los pronombres clíticos en las conversaciones en Facebook Messenger.

\begin{tabular}{|l|l|c|}
\hline Pronombre & Frecuencia absoluta & Frecuencia relativa (\%) \\
\hline Te & 105 & $17.24 \%$ \\
\hline Le & 315 & $51.72 \%$ \\
\hline Les & 70 & $11.49 \%$ \\
\hline Lo & 71 & $10.83 \%$ \\
\hline Los & 28 & $4.59 \%$ \\
\hline La & 23 & $3.77 \%$ \\
\hline Las & 2 & $0.32 \%$ \\
\hline Total & 609 & $100.00 \%$ \\
\hline
\end{tabular}

Tabla 4. Pronombres utilizados por los informantes

Esta tabla presenta todos los casos de los pronombres clíticos en el corpus incluso en los casos de su uso con pronombre de objeto indirecto. Nótese la baja frecuencia de la(s) en el corpus que Lipski (1994) notó como rasgo del español ecuatoriano en la sierra. Aun hablantes monolingües no lo tienen en su repertorio.

Cuando se refiere al leísmo de $3 \underline{a}$ persona en este estudio, se tiene en mente casos como los siguientes. Se ve su uso con referentes masculinos (3), femenino (1), y plural (2). 
Dialectologia 22 (2019), 57-80.

ISSN: 2013-2247

1. Buenas tardes mamá $\mathrm{N}$ sabe que de ladito me emocioné de verle a su hija junto a ustedes quería felicitarles por ser siempre unos padres incondicionales siempre ayudando a sus hijos.

2. Es una alegría tenerles en casa [refiriendo a sus hijos]

3. A lo mejor no le recuerde El vivía en $\mathrm{P}$ después se radico en Azogues. Solo a Zhoray nos íbamos de vez en cuando [refiriendo a su padre]

Cuando se refiere al leísmo de cortesía, se tiene en mente casos como los siguientes. Se puede ver el uso del leísmo de cortesía con referentes singulares y plurales. También se ve su uso con salutaciones y deseos de bienestar que se discutirán más abajo.

4. Y q Dios le derrame siempre de bendiciones

5. Bueno cariño me hoy a merendar ya hablamos en otra oportunidad que diosito les bendiga y sigan unidos en familia

La Tabla 5 demuestra la distribución y frecuencia del uso estándar de $l o(s)$, la(s), el uso de leísmo y el uso de leísmo de cortesía en Facebook Messenger. Se excluyeron los casos del uso de le(s) con pronombres indirectos.

\section{Leísmo}

\begin{tabular}{|l|l|l|}
\hline & Frecuencia absoluta & Frecuencia relativa (\%) \\
\hline Uso estándar de lo(s), la(s) de 3a persona & 67 & $42.67 \%$ \\
\hline Casos de leísmo de 3a persona & 90 & $57.32 \%$ \\
\hline Total de 3a persona & 157 & $100.00 \%$ \\
\hline
\end{tabular}

\section{Leísmo de cortesía}

\begin{tabular}{|l|c|l|}
\hline $\begin{array}{l}\text { Uso estándar de } l o(s), l a(s) \\
\text { de 2a persona }\end{array}$ & 57 & $35.62 \%$ \\
\hline Casos de leísmo de cortesía & 103 & $64.37 \%$ \\
\hline Total de 2a persona & 160 & $100.00 \%$ \\
\hline
\end{tabular}


La tabla nota la variación que se encuentra en el español ecuatoriano en cuanto a los dos patrones del leísmo analizados en este estudio. Se ve que los informantes utilizaron los dos tipos de leísmo con más frecuencia en comparación con la variante estándar. Los informantes utilizaron el leísmo de cortesía con más frecuencia que el leísmo de 3a persona también. El uso del leísmo de cortesía no es una selección marcada en estos resultados, sino que parece ser la preferencia de los informantes. Estos resultados son parecidos a los resultados de un cuestionario hecho por Dumitrescu \& Branza (2012) sobre el uso de leísmo en varias ciudades latinoamericanas y españolas. La tabla abajo presenta los resultados del cuestionario en Quito.

\begin{tabular}{|l|l|l|l|l|l|l|}
\hline Ciudad & \multicolumn{3}{|c|}{ Usted } & \multicolumn{3}{c|}{ Él } \\
\cline { 2 - 7 } & F. abs. le & F. abs. lo & F. rel. le & F. abs. le & F. abs. lo & F. rel. le \\
\hline Quito & 34 & 34 & $50 \%$ & 11 & 58 & $16 \%$ \\
\hline
\end{tabular}

Tabla 5. Leísmo en Quito (Dumitrescu \& Branza 2012: 679)

La tabla demuestra que la preferencia leísta en Azogues no es extraña en el español ecuatoriano de la sierra, especialmente el leísmo de cortesía. Este estudio demuestra datos de conversaciones reales entre hablantes ecuatorianos, confirmando las preferencias demostradas en los cuestionarios de Branza sino en conversaciones naturales entre amigos en Facebook Messenger.

\subsection{Factores lingüísticos}

\subsection{1 +/- animado}

Este estudio considera el factor de [+/- animado] como factor en la selección de pronombres, junto con otros estudios sobre el tema (Palacios 2002). La tabla siguiente presenta los resultados del estudio en cuanto a este asunto. Se excluyeron los casos del uso estándar con referentes de $2^{\mathrm{a}}$ persona y casos de leísmo de cortesía. 
Dialectologia 22 (2019), 57-80.

ISSN: 2013-2247

\begin{tabular}{|l|l|l|l|l|}
\hline & Uso estándar de lo(s), la(s) & Porcentaje & Leísmo & Porcentaje \\
\hline +animado & 16 & $23.88 \%$ & 78 & $86.67 \%$ \\
\hline - animado & 51 & $76.11 \%$ & 13 & $14.45 \%$ \\
\hline Total & 67 & $100.00 \%$ & 90 & $100.00 \%$ \\
\hline
\end{tabular}

Tabla 6. +/-animado

Se observa que el uso de $l o(s)$, la(s) acompaña los referentes [-animados] mientras que el leísmo tiende a acompañar a referentes [+animados].

La mayoría de los casos de leísmo de 3a persona refieren a entes humanos. Sin embargo, hay unos casos cuando refieren a entes no humanos. La siguiente parte de una conversación entre P1 e Informante 29 demuestra un caso del leísmo de cosa que se encuentra infrecuentemente en este corpus de datos.

Inf. 29: Bueno los vi bien y están un poco acabados mejor los donó

P1: [foto de zapatos]

P1: ok

P1: Asi quedaron sus zapatos les pinte

Entonces, los informantes de este estudio prefieren el uso del leísmo con entes humanos y animados sobre el uso de leísmo de cosa. Sin embargo, la mayoría de los casos reflejan el leísmo de persona como en el siguiente, cuando P1 refiere a su esposo.

Está bien le tengo envejeciendo a mi lado

\subsubsection{Género del referente}

La tabla siguiente presenta los casos de los fenómenos estudiados en cuanto al referente. Se divide en dos lados con los casos de leísmo de 3a persona y el uso estándar de 3 a persona y los casos con leísmo de cortesía y el uso estándar de 2 a persona. 
3a persona

2a persona

\begin{tabular}{|l|l|l|l|l|l|l|l|l|l|}
\hline & \multicolumn{3}{|l|}{ Uso estándar } & \multicolumn{2}{l|}{ Leísmo } & \multicolumn{2}{l|}{ Uso estándar } & Leísmo de cortesía & Total \\
\hline $\begin{array}{l}\text { Masculino } \\
\text { singular }\end{array}$ & 38 & $32.2 \%$ & 45 & $38.13 \%$ & 21 & $17.79 \%$ & 14 & $11.86 \%$ & 118 \\
\hline $\begin{array}{l}\text { Femenino } \\
\text { singular }\end{array}$ & 15 & $15.3 \%$ & 21 & $21.42 \%$ & 10 & $10.2 \%$ & 52 & $53.06 \%$ & 98 \\
\hline $\begin{array}{l}\text { Masculino } \\
\text { plural }\end{array}$ & 13 & $14.13 \%$ & 18 & $19.56 \%$ & 24 & $26.08 \%$ & 37 & $40.21 \%$ & 92 \\
\hline $\begin{array}{l}\text { Femenino } \\
\text { plural }\end{array}$ & 1 & $11.12 \%$ & 6 & $66.67 \%$ & 2 & $22.23 \%$ & 0 & 0 & 9 \\
\hline
\end{tabular}

Tabla 7. Género del referente

La distribución de casos de masculino singular es igual entre el uso estándar y el leísmo de 3a persona. Se ve que los informantes utilizan leísmo de cortesía más cuando el referente es femenino.

\subsubsection{Posición de pronombre}

El otro factor lingüístico analizado en este estudio es la posición del pronombre pre-verbal o pos-verbal. La tabla abajo presenta los resultados del estudio en cuanto a este asunto.

Uso estándar

Uso estándar pre-verbal Uso estándar pos-verbal

Total uso estándar

Leísmo pre-verbal

Leísmo pos-verbal

Total leísmo

Leísmo de cortesía pre-verbal

Leísmo de cortesía pos-verbal

Total leísmo de cortesía

Tabla 8. Posición del pronombre
Frecuencia absoluta

Frecuencia relativa 
La tabla demuestra que no hay una diferencia en cuanto al uso de pronombres clíticos en el español ecuatoriano. Los ejemplos siguientes demuestran las dos posiciones oracionales analizadas.

6. Una pregunta le has visto al pastor P quiero conectarme y no se como.

7. Bien bien quiero invitarles para casi carnaval sábado ya hable también con el $\mathrm{P}$ y a la $\mathrm{N}$ y el $\mathrm{M}$ q dices primis

Los ejemplos demuestran un caso de leísmo de 3a persona y otro de leísmo de cortesía.

\subsubsection{Verbos utilizados con leísmo}

En esta sección, se analizan los verbos utilizados con los dos tipos de leísmo de este análisis. Las palabras subrayadas en negritas son las que aparecen con los dos tipos de leísmo.

Verbos utilizados con leísmo de cortesía

- Felicitar

- Saludar

- Llamar

- Invitar

- Recordar

- Tener

- Ver

- Querer

- Amar

- Encontrar

- Proteger

- Demarrar

- lluminar

- Agradecer

- Visitar

- Avisar

- Dejar

- Bendecir

- Esperar
Verbos utilizados con leísmo de persona

- Felicitar

- Saludar

- Llamar

- Invitar

- Recordar

- Tener

- Ver

- Traer

- Hacer

- Apoyar

- Agarrar

- Conocer

- Ayudar

- Bautizar

- Bañar

- Enseñar

- Poner

- Perder

- Pintar 
- Chequear

- Depositar

- Reconocer

- Recibir

- Entender

El leísmo de cortesía es aceptable con verbos que se usan con deseos de bienestar y saludos como los ejemplos siguientes: le saludo, un gusto saludarle, que Dios le proteja, que Dios le ilumine, que Dios le bendiga, que Dios le derrame con muchas bendiciones, etc. Los contextos en los cuales se puede utilizar el leísmo son más restringidos que quiere decir que hay menos posibilidades en cuanto al empleo del leísmo de cortesía. No obstante, los datos de este estudio demuestran que el uso del leísmo de cortesía ha extendido a otros contextos fuera de las fórmulas fijas mencionadas anteriormente. Los contextos en los cuales se puede emplear el leísmo de persona son más amplios. Sin embargo, la frecuencia absoluta del leísmo de cortesía es más grande que la del leísmo de persona. El ejemplo siguiente demuestra un caso del leísmo de cortesía con un verbo de la lista que no refleja un uso fijo formulado.

Me puede ayudar con su numero para el le llame [a usted] y hable como mismo es eso.

\subsection{Factores extralingüísticos}

Debido a los métodos utilizados para recoger datos en este estudio, no se pudo hacer un análisis extendido del efecto de los factores sociales en el uso del leísmo de persona o de cortesía. Para hacer un análisis sociolingüístico lo que se hizo fue excluir los datos de P1 y P2 y solo analizar los datos de sus amigos en Facebook Messenger para evitar una representación sesgada de los resultados.

\subsubsection{Sexo}

La tabla 10 presenta los resultados en cuanto al factor del sexo del informante y al uso de leísmo de 3 a persona y de cortesía. 
cortesía

\begin{tabular}{|l|l|l|l|l|l|l|}
\hline Hombre & 26 & 17.68 & 16 & 10.88 & 38 & 25.85 \\
\hline Mujer & 16 & 10.88 & 24 & 16.32 & 27 & 18.36 \\
\hline
\end{tabular}

Total $147 \quad 100.00 \%$

Tabla 9. Sexo

Se evidencia que hay un aumento en cuanto al número de casos del leísmo de cortesía en los mensajes de los hombres. Como se mencionó anteriormente, se excluyeron los resultados de P1 y P2 para hacer este análisis, sin embargo, sus resultados se encuentran en Tabla 10 para compararlos con los resultados de Tabla 9.

\begin{tabular}{|c|c|c|c|c|c|c|}
\hline & $\begin{array}{l}\text { Uso } \\
\text { estándar }\end{array}$ & $\begin{array}{l}\text { Frecuencia } \\
\text { (\%) }\end{array}$ & Leísmo & $\begin{array}{l}\text { Frecuencia } \\
\text { (\%) }\end{array}$ & $\begin{array}{l}\text { Leísmo de } \\
\text { cortesía }\end{array}$ & $\begin{array}{l}\text { Frecuencia } \\
\text { (\%) }\end{array}$ \\
\hline $\mathrm{P} 1$ & 68 & 40.0 & 47 & 27.64 & 29 & 17.05 \\
\hline P2 & 14 & 8.23 & 3 & 1.76 & 9 & 5.29 \\
\hline
\end{tabular}

Tabla 10. P1 y $\mathrm{P} 2$

P1 es muy activa en Facebook Messenger, entonces sus resultados superan los resultados de P2 que no es tan activa. Hay más casos del leísmo de 3a persona en los mensajes de P1, lo cual se refleja también en los mensajes de sus amigos femeninos; hay un aumento en los casos de leísmo de 3a persona. En los mensajes de P1, hay casos del uso estándar en fórmulas fijas de saludo y despedida como un gusto saludarlo y otros ejemplos de este tipo. Además, P2 tiene maestría, lo cual puede explicar su evitación de formas asociadas con leísmo de 3ạ persona.

\subsubsection{Nivel de educación}

La Tabla 12 presenta los resultados en cuanto al efecto del nivel de educación y su efecto en el uso del leísmo. 


\begin{tabular}{|c|c|c|c|c|c|c|}
\hline & Uso estándar & (\%) & Leísmo & (\%) & \multicolumn{2}{|c|}{$\begin{array}{l}\text { Leísmo de } \\
\text { cortesía }\end{array}$} \\
\hline Primaria & 10 & 6.8 & 16 & 10.88 & 23 & 15.64 \\
\hline Secundaria & 14 & 9.5 & 4 & 2.72 & 21 & 14.28 \\
\hline Universitaria & 18 & 12.24 & 20 & 13.6 & 21 & 14.28 \\
\hline Total & 147 & & 100.00 & & & \\
\hline
\end{tabular}

Se observa la evitación del leísmo de 3 a persona por los informantes con educación secundaria. Sin embargo, no hay dicha evitación en los mensajes de los informantes con educación universitaria. Fernández-Ordóñez (1999) sugiere que los hablantes con más educación tiendan a utilizar más las formas estándares. Los resultados de este estudio sugieren que los hablantes con educación secundaria eviten el leísmo a favor de formas más estándares, probablemente para evitar asociación con los hablantes con educación primaria. Los hablantes en el medio quieren diferenciarse del grupo con educación primaria, aunque los hablantes con educación universitaria no tienen ese perjuicio hacia el leísmo de 3ạ persona en sus mensajes. El leísmo de cortesía no parece tener esta asociación según los resultados de este estudio. P1 refleja el patrón de hablantes con educación universitaria por no mostrar esta evitación del leísmo de 3a persona.

\section{Discusión y conclusión}

Este estudio ha analizado dos patrones del leísmo en el español de Azogues, Ecuador. Ha investigado el papel de factores lingüísticos y sociales en cuanto a su uso. Este estudio enfocó en su uso en el español monolingüe de Ecuador. Este estudio también añade al conocimiento sobre el uso del leísmo en el español ecuatoriano. El leísmo de cortesía y de 3 a persona ocurren con más o menos la misma frecuencia en los resultados. El leísmo de cortesía ocurre en más casos que el uso estándar de 2a persona.

Los factores lingüísticos que afectan al uso del leísmo son el factor de [+/animado] para el leísmo de 3 a persona. El leísmo acompaña a referentes animados, mientras que el uso estándar de $3 \underline{a}$ persona acompaña referentes no animados. El 
leísmo de cortesía acompaña a referentes femeninos singulares y masculinos plurales con más frecuencia. Sin embargo, no hay diferencias observables en cuanto al comportamiento de los dos patrones de leísmo en cuanto a su preferencia posicional en relación con el verbo de la oración; los dos tienen una preferencia pre-verbal en los resultados de este estudio. Los informantes de este estudio todavía utilizan la(s) aunque con una frecuencia muy baja. El leísmo de cortesía parece reemplazar la en este contexto, mientras que no reemplaza el uso de lo de 2 a persona (con usted). Los informantes de este estudio tienen un sistema pronominal que distingue caso pero que tiene tendencias leístas. El uso de leísmo de cortesía es muy frecuente y parece no ser marcado.

En cuanto al análisis de los factores sociales, solo se pudo hacer un análisis restringido debido a la naturaleza de los datos recogidos para este estudio. Sin embargo, se encontró que hay más casos del leísmo de cortesía en los datos de informantes masculinos. También, en cuanto al efecto de la educación, se ve que el leísmo de 3 a persona es marcado a causa de su evitación por los informantes con educación secundaria, una estrategia empleada por hablantes en el medio para evitar asociación con los hablantes con educación primaria. García \& Otheguy (1983) notan que el sistema completo (estándar) tiene más prestigio en el español de Ecuador. Los datos de este estudio sugieren que sean los hablantes con educación secundaria que quieren alejarse de los rasgos asociados con los que solo tienen educación primaria. Sin embargo, los informantes con educación universitaria no demuestran evitación del leísmo.

La importancia de la cortesía en cuanto al leísmo de cortesía es importante cuando se analizan todos los contextos en los cuales aparece y los verbos que lo acompañan. El uso de face flattering acts en los datos es frecuente debido al número de conversaciones analizadas que se ve en su uso con fórmulas fijas de saludo y despedida y con deseos de bienestar del tipo, Que Dios le bendiga, etc. Se puede notar la importancia de la relación entre locutor e interlocutor y la cortesía que utilizan por la inclusión de tantos deseos de bienestar y saludos en las conversaciones. Cuando utilizan este tipo de deseo o saludo hay una opción entre la selección del uso estándar, lo(s), la(s) o el leísmo de cortesía. Parece que la segunda opción es la que gana en esta lucha 
según los resultados de este estudio. Sin embargo, este leísmo se comporta como el leísmo de 3ạ persona en cuanto a su preferencia posicional en la oración.

El leísmo de cortesía es un "cambio en progreso" (Dumitrescu \& Branza 2012) que merece más atención para entender su uso en el español actual. Este estudio ha investigado sobre este fenómeno en el español de Azogues, Ecuador por el uso de datos de Facebook Messenger, una fuente nueva para recoger datos lingüísticos en el estudio del español actual.

\section{Referencias}

Albelda Marco, M. (2008) "El refuerzo de la imagen social en conversaciones coloquiales en español peninsular: La intensificación como categoría pragmática", en D. Bravo (ed.), Estudios de la (des)cortesía en español: Categorías conceptuales y aplicaciones a corpora orales y escritos, Estocolmo-Buenos Aires: Edice/Editorial Dunken, 93-118.

BranzA, Mircea-Doru (2009a) "Dependencia contextual en el uso variable de los clíticos de 3a persona", en R. Utale (ed.), Come devozione come durevole, Studi in onore della Professa Doina Derer, Bucarest: Editura Universitatii din Bucuresti, 51-64.

BranZA, Mircea-Doru (2009b) "Un experimento de cambio lingüístico. El leísmo en España e Hispanoamérica: divergencias y convergencias", Analele Universitatii din Bucuresti, Limbi si literatura strasine 58(2), 71-97.

BRANZA, Mircea-Doru (2011) “Norma y 'antinorma' en el español estándar de España e Hispanoamérica: el caso del leísmo masculino y femenino de persona", en Actas de la Conferencia Internacional, El otro- lo otro - la otredad, Bratislava 21-23 de octubre de 2009, 36-47.

BÜTTNER, Thomas (1993) Uso de quichua y del castellano en la sierra ecuatoriana, Quito: Ediciones Abya-Yala.

CALDERÓn CAMPOS, Miguel (2010) “Formas de tratamiento", en Milagros Aleza Izquierdo \& José María Enguita Utrilla (coords.), La lengua española en América: normas y usos actuales, València: Universitat de València.

Cantero Sandoval, G. (1979) "Casos de leísmo en México", Anuario de Letras: Revista de la Facultad de Filosofía y Letras 17, 305-308. 
Dialectologia 22 (2019), 57-80.

ISSN: 2013-2247

Caravedo, Rocío (1996) "Pronombres objeto en el español andino", Anuario de lingüística hispánica, 7, 2, 545-568.

CRYSTAL, David (2006) Language and the Internet, New York: Cambridge University Press.

Demello, G. (2002) "Leísmo in Contemporary Spanish American Educated Speech", Linguistics $40,2,261-283$

DUMITRESCU, Domnita (2011) “Cortesía codificada versus cortesía interpretada en español: consideraciones generales", Glosas 7, 2, 1-12.

DumitresCu, Domnita \& Mircea-Doru Branza (2012) “Sobre el llamado 'leísmo de cortesía' en Hispanoamérica", en, Julio Escamilla Morales \& Grandfield Henry Vega (eds.), Miradas multidisciplinarias a los fenómenos de cortesía y descortesía en el mundo hispánico, Barranquilla-Estocolmo: Universidad del Atlántico y Programa EDICE.

FernÁNDEZ-ORDóÑEZ, Inés (1999) "Leísmo, laísmo y loísmo", en Ignacio Bosque \& Violeta Demonte (dirs.), Gramática Descriptiva de la Lengua Española, Madrid, Espasa Calpe, vol. I, 1317-1397.

FLoRES MeJía, Esthela (2014) “Actitudes lingüísticas en Ecuador. Una tradición normativa que subsiste", en Ana Beatriz Chiquito, \& Miguel Ángel Quesada Pacheco (eds.), Actitudes lingüísticas de los hispanohablantes hacia el idioma español y sus variantes, Bergen Language and Linguistic Studies (BeLLS), 5, 404-488.

García Charola, Érica \& Ricardo L. Otheguy (1983) "Being polite in Ecuador: Strategy reversal under language contact", Lingua 61, 103-132.

GodenZZI, Juan Carlos (1986) "Pronombres de objeto directo e indirecto del castellano en Puno", Lexis 10, 2, 187-201.

Gómez TORREGO, Leonardo (2006) Hablar y escribir correctamente, Madrid: Arco Libros.

HABOUD, Marleen (1998) Quichua y castellano en los andes ecuatorianos: Los efectos de un contacto prolongado, Ediciones: Abya-Yala.

KERBRAT-ORECCHIONI, Catherine (1997) "A multilevel approach in the study of talk-in-interaction", Pragmatics 7, 1, 1-20.

KLEE, Carol A. (1990) "Spanish-Quechua language contact: The clitic pronoun system in Andean Spanish", Word 41, 1, 35-46.

LIPSKI, John (1994) Latin American Spanish, New York: Longman Group Limited.

LoRenzo RAMOS, A. (1981) "Algunos datos sobre el leísmo en el español de Canarias", en M. Alvar (coord.), Actas del 1er Simposio Internacional de Lengua Española, 1978, Las Palmas: Ediciones del Excmo. Cabildo Insular de Gran Canaria, 175-180. 
LORENZO RAMOS, A. (1984) "Observaciones sobre el uso de los pronombres en el español de Canarias", en M. Alvar (coord.), Actas del 2 o Simposio Internacional de Lengua Española, 1981, Las Palmas: Ediciones del Excmo. Cabildo Insular de Gran Canaria, 253-264.

MUYSKEN, Pieter (1985) "Contactos entre quechua y castellano en el Ecuador", en Segundo Moreno, Memorias del primer simposio europeo sobre antropología del Ecuador, Quito, Ecuador: Ediciones Abya, 377-452.

OLSEN, Michael K. (2015) "Current trends among Ecuadorian dialects: Insights from Internet interviews", Dialectologia 14, 75-96 <http://www.publicacions.ub.edu/ revistes/dialectologia14/>.

Palacios Alcaine, Azucena (2002) "Leísmo y loísmo en el español ecuatoriano: el sistema pronominal del español andino", en, Homenaje al Dr. Luis Jaime Cisneros, vol. 1, Lima: Pontificia Universidad Católica del Perú, 389-408.

PAlacios Alcaine, Azucena (2005) "La influencia del quichua en el español andino ecuatoriano", en C. Ferrero Pino y N. Lasso von Land (eds.), Variedades lingüísticas y lenguas en contacto en el mundo de habla hispana, Brooks Library: Bloomington, 44-52.

Real ACAdemia EsPañola/ Asociación de ACAdemias de la lengua eSPañola (2009) Nueva Gramática de la lengua española, Tomo I: Morfología, Sintaxis I, tomo II: Sintaxis II, Madrid: Espasa Libros, S.L.

SebBA, Mark (2012) "Researching and theorizing multilingual texts", en Mark Sebba, Shahrzad Mahootian \& Carla Jonsson (eds.), Language Mixing and Code-Switching in Writing: Approaches to Mixed-Language Written Discourse, New York: Routledge, 2-26.

VAquero de Ramírez, María (1996) El español de América, v. 1 y 2, Madrid: Arco Libros. 